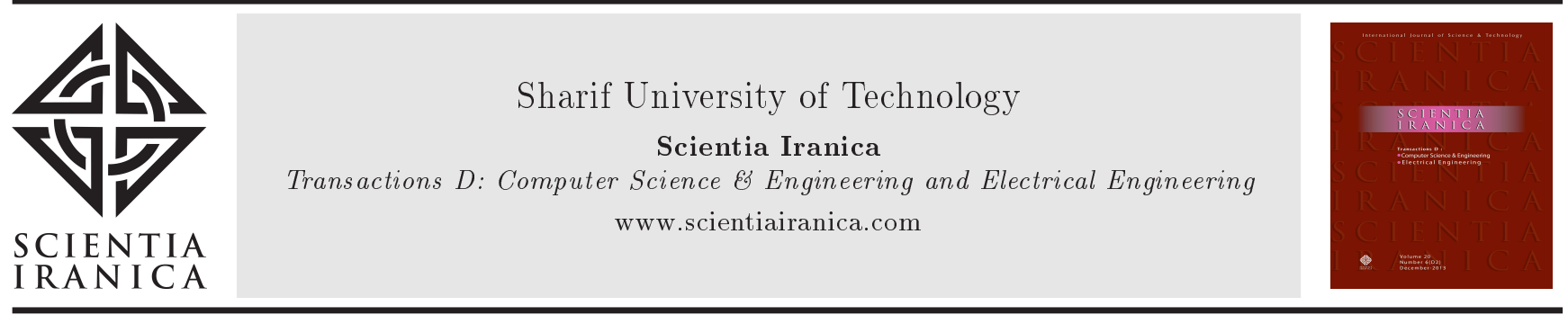

\title{
Nonsingular fast terminal sliding-mode stabilizer for a class of uncertain nonlinear systems based on disturbance observer
}

\author{
S. Mobayen ${ }^{\mathrm{a}, *}$ and F. Tchier ${ }^{\mathrm{b}}$ \\ a. Department of Electrical Engineering, Faculty of Engineering, University of Zanjan, Zanjan, Iran. \\ b. Department of Mathematics, King Saud University, P.O. Box 22452, Riyadh 11495, Saudi Arabia. \\ Received 7 November 2015; received in revised form 17 April 2016; accepted 24 May 2016
}

\author{
KEYWORDS \\ Finite-time stabilizer; \\ Nonsingular fast \\ terminal sliding mode; \\ Third-order system; \\ Disturbance observer; \\ Robustness.
}

\begin{abstract}
This paper investigates a novel nonsingular fast terminal sliding-mode control method for the stabilization of the uncertain time-varying and nonlinear thirdorder systems. The designed disturbance observer satisfies the finite-time convergence of the disturbance approximation error and the suggested finite-time stabilizer assures the presence of the switching behavior around the switching curve in the finite time. Furthermore, this approach can overcome the singularity problem of the fast terminal sliding-mode control technique. Moreover, knowledge about the upper bounds of the disturbances is not required and the chattering problem is eliminated. Usefulness and effectiveness of the offered procedure are confirmed by numerical simulation results.
\end{abstract}

(C) 2017 Sharif University of Technology. All rights reserved.

\section{Introduction}

\subsection{Background and motivations}

The rigorous foundation for the theory of finite-time stabilization was first presented by Bhat and Bernstein [1]. Sliding-Mode Control (SMC) is efficiently applied for the stabilization and controller design of various linear and nonlinear dynamical systems such as singular systems [2], robotic manipulators [3], nonholonomic mobile robots [4,5], fractional order systems [6], chaotic systems [7,8], under-actuated systems [9], etc. SMC is an influential control technique, which has access to the favorite responses in spite of the uncertainties and disturbances [10,11]. The important features of SMC technique are robustness against parametric uncertainties, superior transient performance, quick responses, insensitivity to the bounded external disturbances, and computational simplicity compared

\footnotetext{
*. Corresponding author. Tel.: +982433054208

E-mail address: mobayen@znu.ac.ir (S. Mobayen)
}

to other robust control techniques [12]. The process of SMC design is divided into two phases: (a) the sliding phase, and (b) the reaching phase. Firstly, in sliding phase, a switching curve is specified such that the controlled system displays favorable dynamic performance during the sliding mode [13]. Secondly, in reaching phase, a sliding control law is employed for the state trajectories of the controlled system to converge on the sliding curve. Due to the effect of the sliding curve on the stabilization and transient response of the controlled system, the design procedure of the switching curve is the most important subject in the sliding method [14]. SMC utilizes a discontinuous controller to drive the state trajectories to a predesigned switching surface on which the desired performance besides stability of the system can be obtained [15]. Young et al. [13] proposed SMC observer scheme with only the discontinuous term being fed back through a suitable gain. Generally, traditional SMC has some important problems, such as discontinuous control, which often yields chattering phenomenon [16]. 
Also, it is required in [14] that the external disturbance should be matched, that is, act in the channels of the inputs. To cope with these problems and attain higher accuracy, some new forms of SMC have been proposed. Moreover, SMC cannot guarantee the converging performance of the state trajectories to the origin in the finite time. To tackle the mentioned problem, the Terminal Sliding-Mode Control (TSMC) procedure has been suggested and performed in several control systems. TSMC technique proposes some excellent specifications such as rapid response and finite-time stability in comparison with linear SMC [17]. TSMC is principally suitable for high-precision stabilization and control as it precipitates the convergence rate near the origin [18]. However, when the state trajectories of the system are far away from the origin, TSMC cannot present appropriate convergence efficiency like SMC [19]. The Fast Terminal Sliding-Mode Control (FTSMC) idea guarantees fast transient convergence and strong robustness [20]. In the last decade, there has been more attention to the utilization of the mentioned technique for various control problems [21]. Nevertheless, it should be mentioned that the FTSMC technique still requires to be further considered in robustness performances to tackle the system disturbances.

\subsection{Literature review}

In most of the considered research, the boundaries of the perturbations are directly employed in the design of the TSMC law $[22,23]$. To estimate the disturbances, various design procedures based on the disturbance observer have been planned in the recent years [24]. In [25], SMC has been established for the control and stabilization of uncertain and nonlinear dynamical systems using the disturbance observers. In [26], a novel multiple-surface SMC is recommended for the uncertain nonlinear systems and a disturbanceobserver-based approach is defined to estimate the mismatched uncertainties of the system. In [27], the design procedure of the adaptive finite reaching time controllers for the first- and second-order dynamical systems with perturbations is investigated, where the suggested controllers are continuous and retain robustness to the disturbances. The combination of FTSMC and Global Sliding-Mode (GSM) surface for the robust tracking control of nonlinear second-order systems with time-varying uncertainties is investigated in [28]. A recursive FTSMC technique for tracking control of nonholonomic systems in the chained form is proposed in [29], where the tracking errors are allowed to decay to the origin in the finite time with an exponential decay rate. A disturbance-observer-based recursive TSMC tracker is presented in [30] for the finite-time tracking control of third-order non-holonomic systems with unknown external disturbances. An adaptive FTSMC technique combined with GSM scheme is suggested in [31] for the tracking problem of uncertain nonlinear third-order systems. A Linear Matrix Inequalities (LMI)-based second-order FTSMC method is investigated in [32] for the tracking control of nonlinear uncertain systems with matched and mismatched uncertainties. However, the singularity problem is not fully resolved in [26-30]. In [33], the robust synchronization problem of disturbed chaotic systems is investigated, where, using an LMI-based disturbance observer, the boundedness conditions of disturbance errors are satisfied. In $[22,34]$, the disturbance observers are applied to estimate the disturbances and some robust control approaches are considered using the outputs of the disturbance observers. To satisfy the approach of the approximation errors to the origin in the finite time, TSMC disturbance observer has been established in $[20,35,36]$. In [33,34], composite control design procedures of the disturbance-observer-based controller and TSMC are offered for the uncertain structural and nonlinear systems where the proposed disturbance observers are based on the regional pole placement and D-stability theories. In [37], by combining TSMC and second-order SMC approaches, a nonlinear robust control technique and a disturbance observer are designed for the longitudinal dynamics of hypersonic vehicles with uncertainties and disturbances, which can provide high-precision and fast convergence.

\subsection{Contributions}

To the best of our knowledge, there are two disadvantages of TSMC and FTSMC, which are the singularity problem and the requirement of the bounds of the disturbances. In the recent years, very little attention has been paid to both these problems, which is still open in the literature. In this study, based on the disturbance observer presented in [20], we apply a new Nonsingular Fast Terminal Sliding-Mode Control (NFTSMC) approach for the finite-time control of uncertain and nonlinear third-order systems with external disturbances. The singularity problem of FTSMC method is solved by the designed NFTSMC and the disturbance observer is developed to quickly force the disturbance approximation errors to converge to the origin in a finite time.

\subsection{Paper organization}

The structure of the paper is as follows: The formulation of the problem is described in Section 2 . In Section 3, the proposed control mechanism and stability analysis are introduced. Simulation results of the application of the offered disturbance-observerbased NFTSMC method on an uncertain nonlinear third-order system and an uncertain chaotic system are obtained in Section 4. Finally, Section 5 draws the concluding remarks of this research. 


\section{Problem description}

Consider the uncertain nonlinear system as follows:

$$
\begin{aligned}
\dot{x}_{1}= & x_{2}, \\
\dot{x}_{2}= & x_{3}, \\
\dot{x}_{3}= & f(x, t)+\Delta f(x, t)+(b(x, t)+\Delta b(x, t)) u \\
& \quad+d_{0}(x, t), \\
y= & x_{1},
\end{aligned}
$$

where $x=\left[x_{1}, x_{2}, x_{3}\right]^{T}$ is the state vector; $u$ is the control signal; $y$ is the system output; $b(x, t)$ and $f(x, t)$ are the bounded nonlinear functions; and $\Delta b(x, t)$, $\Delta f(x, t)$, and $d_{0}(x, t)$ are the nonlinear functions representing the uncertainties and external disturbances. Furthermore, it is supposed that a positive constant value $\delta$, which is the lower bound of $b(x, t)$, is defined, that is, $\delta=\inf (|b(x, t)|)$. Defining $d(x, t)=\Delta f(x, t)+$ $\Delta b(x, t) u+d_{0}(x, t)$, one can obtain System (1) as:

$$
\begin{aligned}
& \dot{x}_{1}=x_{2}, \\
& \dot{x}_{2}=x_{3}, \\
& \dot{x}_{3}=f(x, t)+b(x, t) u+d(x, t), \\
& y=x_{1} .
\end{aligned}
$$

In order to examine the stabilization problem of the uncertain system, we can rewrite Dynamics (2) in the following form:

$$
\dot{x}=A x+B\{f(x, t)+b(x, t) u+d(x, t)\},
$$

where:

$$
A=\left[\begin{array}{lll}
0 & 1 & 0 \\
0 & 0 & 1 \\
0 & 0 & 0
\end{array}\right], \quad B\left[\begin{array}{l}
0 \\
0 \\
1
\end{array}\right] .
$$

Now, based on the pole-placement procedure, a term $\bar{k} x$ with $\bar{k}=\left\lfloor\bar{k}_{0}, \bar{k}_{1}, \bar{k}_{2}\right\rfloor$ is considered, where $\bar{k}_{i}$ 's are selected such that the deterministic equation $S^{3}+$ $\bar{k}_{2} S^{2}+\bar{k}_{1} S+\bar{k}_{0}=0$ is stable system. Hence, the exponentially stable dynamics are achieved as:

$$
\dddot{x}+\bar{k}_{2} \ddot{x}+\bar{k}_{1} \dot{x}+\bar{k}_{0} x=0,
$$

which designates that $x$ converges to zero. Consequently, System (3) is modified as:

$$
\dot{x}=(A-B \bar{k}) x+B(\bar{k} x+f(x, t)+b(x, t) u+d(x, t)),
$$

where the control input can be considered by the following transformation:

$$
u=b(x, t)^{-1}\{\bar{v}-f(x, t)\},
$$

where $\bar{v}$ is a new control input. The control signal (Eq. (6)) contains two portions: one portion is $-b(x, t)^{-1} f(x, t)$, which is employed to remove the system nonlinearities, and the other portion is $b(x, t)^{-1} \bar{v}$, which is used to weaken the impacts of the perturbations. Substituting Eq. (6) into Eq. (5) gives:

$$
\dot{x}=(A-B \bar{k}) x+B(\bar{v}+\bar{H}),
$$

where $\bar{H}=\bar{k} x+d(x, t)$. The function $d(x, t)$ is a continuous function and, hence, $\bar{H}$ is also continuous. Then, System (7) is completely controllable and can be controlled by several robust control procedures.

Remark 1. In the case that $b(x, t)$ is not invertible, similar to the methods planned in control papers such as [38], one can use the term $b(x, t)^{+}=$ $\left(b(x, t)^{T} b(x, t)\right)^{-1} b(x, t)^{T}$ as the pseudo-inverse of $b(x, t)$.

Lemma 1. Consider the candidate positive-definite functional $V(t)$, which fulfills a differential inequality, as [19]:

$$
\dot{V}(t) \leq-\alpha V(t)-\beta V(t)^{\eta} \quad \forall t \geq t_{0}, V\left(t_{0}\right) \geq 0,
$$

where $\alpha$ and $\beta$ are two positive coefficients, and $\eta$ is a fraction of two odd positive numbers with $1>\eta>0$. As a result, for certain time, $t_{0}$, the above-mentioned function, $V(t)$, approaches the origin in the finite time as [29]:

$$
t_{s}=t_{0}+\frac{1}{\alpha(1-\eta)} \ln \left(\frac{\alpha V\left(t_{0}\right)^{1-\eta}+\beta}{\beta}\right) .
$$

Proof. If two sides of Inequality (8) are divided to $V^{\eta}(t)$, we obtain:

$$
V(t)^{-\eta} \dot{V}(t) \leq-\alpha V(t)^{1-\eta}-\beta
$$

and thus:

$$
d t \leq\left(\frac{-V(t)^{-\eta}}{\alpha V(t)^{1-\eta}+\beta}\right) d V(t)
$$

Integrating two sides of Relation (11) from $t_{0}$ to $t_{s}$ yields:

$$
\begin{aligned}
t_{s} & -t_{0} \leq \int_{V\left(t_{0}\right)}^{0}\left(\frac{V(t)^{-\eta}}{\alpha V(t)^{1-\eta}+\beta}\right) d V(t) \\
& =-\frac{1}{\alpha(1-\eta)}\left[\ln (\beta)-\ln \left(\alpha V\left(t_{0}\right)^{1-\eta}+\beta\right)\right] \\
& =\frac{1}{\alpha(1-\eta)} \ln \left(\frac{\alpha V\left(t_{0}\right)^{1-\eta}+\beta}{\beta}\right),
\end{aligned}
$$

which completes the proof of the lemma. 


\section{Main results}

The auxiliary variable of the sliding disturbance observer can be described as:

$$
s=z-x_{3},
$$

with $z$, which is specified by:

$$
\begin{aligned}
\dot{z}= & -k s-\beta \operatorname{sgn}(s)-\varepsilon s^{p_{0} / q_{0}}-|f(x, t)| \operatorname{sgn}(s) \\
& +b(x, t) u,
\end{aligned}
$$

where $q_{0}$ and $p_{0}$ are odd positive integers with $p_{0}<q_{0}$. The design coefficients $k, \beta$, and $\varepsilon$ are some positive constants and the condition $\beta \geq|d(x, t)|$ is obtained. The TSMC disturbance estimator $\hat{d}$ is specified as:

$$
\hat{d}=-k s-\beta \operatorname{sgn}(s)-\varepsilon s^{p_{0} / q_{0}}-|f(x, t)| \operatorname{sgn}(s)-f(x, t) .
$$

Theorem 1. Consider the disturbed third-order system (Eq. (1)) and TSMC disturbance observer given by Eqs. (13)-(15). Then, the disturbance approximation error of the suggested TSM disturbance observer converges to the origin in the finite time.

Proof. Considering Eqs. (1) and (14) and differentiating Eq. (13), one achieves:

$$
\begin{aligned}
\dot{s}=\dot{z} & -\dot{x}_{3}=-k s-\beta \operatorname{sgn}(s)-\varepsilon s^{p_{0} / q_{0}} \\
& -|f(x, t)| \operatorname{sgn}(s)-f(x, t)-d(x, t) .
\end{aligned}
$$

Construct the candidate Lyapunov function as:

$$
V(s)=\frac{1}{2} s^{2} .
$$

The time derivative of $V(s)$ is given by:

$$
\begin{aligned}
\dot{V}(s)= & s \dot{s}=s\left(-k s-\beta \operatorname{sgn}(s)-\varepsilon s^{p_{0} / q_{0}}-|f(x, t)| \operatorname{sgn}(s)\right. \\
& -f(x, t)-d(x, t)) \leq-k s^{2}-\varepsilon s^{\left(p_{0}+q_{0}\right) / q_{0}} \\
& -\beta|s|+|s||d(x, t)|-|f(x, t)||s|-s f(x, t) \\
& \leq-k s^{2}-\varepsilon s^{\left(p_{0}+q_{0}\right) / q_{0}} \\
& \leq-2 k V(s)-2^{\left(p_{0}+q_{0}\right) / 2 q_{0}} \\
& \varepsilon V(s)^{\left(p_{0}+q_{0}\right) / 2 q_{0}},
\end{aligned}
$$

where based on Lemma 1 and Eq. (18), one obtains that the auxiliary variable $s$ can converge to the origin in the finite time. Consequently, the error $\tilde{d}$ is obtained using Eqs. (1), (13), and (15) as:

$$
\begin{aligned}
\tilde{d}= & \hat{d}-d(x, t)=-k s-\beta \operatorname{sgn}(s)-\varepsilon s^{p_{0} / q_{0}} \\
& -|f(x, t)| \operatorname{sgn}(s)-f(x, t)-d(x, t)=-k s \\
& -\beta \operatorname{sgn}(s)-\varepsilon s^{p_{0} / q_{0}}-|f(x)| \operatorname{sgn}(s)-\dot{x}_{3} \\
& +b(x, t) u=\dot{z}-\dot{x}_{3}=\dot{s} .
\end{aligned}
$$

It clarifies that according to the finite-time convergence of the switching surface, $s$, to the origin, the approximation error, $\tilde{d}$, reaches zero in the finite time. $\square$

In order to develop FTSMC stabilizer using the disturbance observer, the following switching surface is proposed:

$$
\sigma=\dot{s}+\lambda s+\mu s^{\eta}
$$

where $\lambda$ and $\mu$ are two positive coefficients and $\eta$ is a fraction of two odd positive integer numbers with $1>\eta>\frac{1}{2}$.

In order to assure that FTSMC curve approaches zero in the finite time and system states quickly converge to zero, the following theorem is proposed.

Theorem 2. The uncertain third-order system (Eq. (2)) is considered. If the control law is employed as:

$$
\begin{aligned}
\dot{u}= & b(x, t)^{-1}\left(\kappa \operatorname{sgn}(\sigma)|\sigma|^{\eta}+\gamma \sigma+\left(\lambda+\mu \eta s^{\eta-1}\right) \dot{s}\right. \\
& +\ddot{z}-\dot{f}(x, t)-\dot{b}(x, t) u+\ddot{s}-\dot{\hat{d}}),
\end{aligned}
$$

where $\gamma$ and $\kappa$ are two arbitrary positive coefficients, then the trajectories of Eq. (2) are enforced to move to the switching curve (Eq. (13)) in the finite time and to stay on it.

Proof. The candidate Lyapunov function is given as:

$$
V(\sigma)=\frac{1}{2} \sigma^{T} \sigma .
$$

From Eqs. (2), (13), and (20), the derivative of $\sigma$ can be obtained as:

$$
\begin{aligned}
\dot{\sigma}= & \ddot{s}+\lambda \dot{s}+\mu \eta s^{\eta-1} \dot{s}=\left(\lambda+\mu \eta s^{\eta-1}\right) \dot{s}+\ddot{z}-\dot{f}(x, t) \\
& -\dot{b}(x, t) u-b(x, t) \dot{u}-\dot{d}(x, t) .
\end{aligned}
$$

Differentiating $V(\sigma)$ and using Eq. (23), one can find:

$$
\begin{aligned}
\dot{V}(\sigma)= & \sigma^{T} \dot{\sigma}=\sigma^{T}\left(\left(\lambda+\mu \eta s^{\eta-1}\right) \dot{s}+\ddot{z}-\dot{f}(x, t)\right. \\
& -\dot{b}(x, t) u-b(x, t) \dot{u}+\ddot{s}-\dot{\hat{d}}),
\end{aligned}
$$

where, substituting Eq. (21) in Eq. (24), we obtain: 


$$
\begin{aligned}
\dot{V}(\sigma) & =-\sigma^{T} \kappa \operatorname{sgn}(\sigma)|\sigma|^{\eta}-\sigma^{T} \gamma \sigma \\
& =-\gamma|\sigma|^{2}-\kappa|\sigma|^{\eta+1} \\
& =-\alpha V(\sigma)-\beta V^{\bar{\eta}}(\sigma),
\end{aligned}
$$

where $\bar{\eta}=(\eta+1) / 2<1, \alpha=2 \gamma>0$ and $\beta=2^{\bar{\eta}} \kappa>$ 0 . Then, the Lyapunov function (Eq. (22)) converges gradually to zero and the switching curve approaches zero in the finite time.

Now, considering the FTSMC law demonstrated in Eq. (21), the term $\mu \eta s^{\eta-1} \dot{s}$ may lead to a singularity problem if $\dot{s} \neq 0$ when $s=0$ due to the negative power of $s$. Thus, the FTSMC cannot satisfy a bounded control action if $\dot{s} \neq 0$ when $s=0$. Then, the NFTSMC is suggested to overcome the singularity phenomenon of the FTSMC.

Define the NFTSMC stabilizer based on the disturbance observer as follows:

$$
\sigma=s+\frac{1}{\mu}(\dot{s}+\lambda s)^{\frac{1}{\eta}}
$$

In order to dominate the singularity phenomenon of the FTSMC and guarantee the finite-time convergence of the state trajectories to zero, the following theorem is proposed.

Theorem 3. The uncertain and nonlinear thirdorder system (Eq. (2)) is considered. Applying the NFTSMC law:

$$
\begin{aligned}
\dot{u}= & b(x, t)^{-1}\left(\mu \eta(\dot{s}+\lambda s)^{1-\frac{1}{\eta}}\left(\kappa \operatorname{sgn}(\sigma)|\sigma|^{\eta}+\gamma \sigma\right)\right. \\
& +\ddot{z}-\dot{f}(x, t)-\dot{b}(x, t) u+\lambda \dot{s}+\ddot{s}-\dot{\hat{d}}),
\end{aligned}
$$

with some positive coefficients $\gamma$ and $\kappa$, the states of Eq. (2) are forced to move from the initial conditions to the switching curve (Eq. (13)) in the finite time and to stay on it.

Proof. The Lyapunov function is considered as:

$$
V(\sigma)=\frac{1}{2} \sigma^{T} \sigma
$$

From Eqs. (2), (13), and (26), the derivative of $\sigma$ can be found as:

$$
\begin{aligned}
\dot{\sigma}= & \dot{s}+\frac{1}{\mu \eta}(\ddot{s}+\lambda \dot{s})(\dot{s}+\lambda s)^{\frac{1}{\eta}-1}=\dot{s} \\
& +\frac{1}{\mu \eta}(\ddot{z}-\dot{f}(x, t)-\dot{b}(x, t) u-b(x, t) \dot{u} \\
& -\dot{d}(x, t)+\lambda \dot{s})(\dot{s}+\lambda s)^{\frac{1}{\eta}-1} .
\end{aligned}
$$

Differentiating $V(\sigma)$ and using Eq. (29), we obtain:

$$
\begin{aligned}
\dot{V}(\sigma)= & \sigma^{T} \dot{\sigma}=\sigma^{T}\left(\dot{s}+\frac{1}{\mu \eta}(\ddot{z}-\dot{f}(x, t) u\right. \\
& \left.-\dot{b}(x, t) \dot{u}+\lambda \dot{s}+\ddot{s}-\dot{\hat{d}})(\dot{s}+\lambda s)^{\frac{1}{\eta}-1}\right),
\end{aligned}
$$

where, substituting Eq. (27) in Eq. (30), one can achieve:

$$
\begin{aligned}
\dot{V}(\sigma) & =-\sigma^{T} \kappa \operatorname{sgn}(\sigma)|\sigma|^{\eta}-\sigma^{T} \gamma \sigma=-\gamma|\sigma|^{2}-\kappa|\sigma|^{\eta+1} \\
& =-\alpha V(\sigma)-\beta V^{\bar{\eta}}(\sigma),
\end{aligned}
$$

where $\bar{\eta}=(\eta+1) / 2<1, \alpha=2 \gamma>0$, and $\beta=2^{\bar{\eta}} \kappa>0$. Thus, the NFTSMC surface converges to the origin in the finite time and the states of the system quickly converge to zero. $\square$

Since the discontinuous switching function $\operatorname{sgn}($. shown in Eqs. (21) and (27) can result in chattering problem, undesired responses can occur in the nonlinear third-order system. To avoid this problem, the function $\operatorname{sgn}($.$) can be replaced by the following$ continuous saturation function:

$$
\operatorname{sat}(\sigma)= \begin{cases}\operatorname{sgn}(\sigma), & |\sigma|>\Phi \\ \frac{\sigma}{\Phi}, & |\sigma| \leq \Phi\end{cases}
$$

where $\Phi$ is the boundary-layer thickness. Furthermore, although the existence of the proposed NFTSMC can be guaranteed outside $\Phi$, it cannot be satisfied inside $\Phi$. In the worst situation, the state trajectories of the system would only reach $\Phi$. This will considerably influence the steady-state characteristics of the system.

Remark 2. From Eq. (31), the derivative of the Lyapunov function is negative semi-definite and guarantees that $V(\sigma)$ and $\sigma$ are bounded. It is deduced from Eq. (20) that $s$ and $\dot{s}$ are two bounded functions. Since $V(0)$ is a bounded scalar and $V(\sigma)$ is non-increasing, it can be concluded that $\lim _{t \rightarrow \infty} \int_{0}^{t}\|\sigma\| d t$ and $\lim _{t \rightarrow \infty} \int_{0}^{t}\|s\| d t$ are also bounded. Thus, according to Barbalat's lemma and based on the boundedness of $\lim _{t \rightarrow \infty} \int_{0}^{t}\|s\| d t$ and $\dot{s}$, the auxiliary variable $s$ converges asymptotically to zero, that is, $\lim _{t \rightarrow \infty} \int_{0}^{t} s d t=0$.

Remark 3. In the situations that some of the variables such as:

$$
\ddot{z}, \quad \ddot{s}, \quad \dot{\hat{d}}, \quad \text { and } \quad \dot{f}(x, t)
$$

are not measurable, one can employ the delayedfeedback control method to model these variables. Specially, the delay term in the form of Euler approximation of the derivative function can be applied for the derivatives of the variables [31]. The variable $\ddot{z}$ is replaced by a delay function, $\ddot{z}=\frac{1}{h}[\dot{z}(t)-\dot{z}(t-h)]$, if the delay $h>0$ is sufficiently small. 


\section{Simulation results}

Example 1. Consider the following uncertain nonlinear third-order system [39]:

$$
\begin{aligned}
& \dot{x}_{1}=x_{2}, \\
& \dot{x}_{2}=x_{3}, \\
& \dot{x}_{3}=-3 x_{3}-4 x_{2}-2 x_{1}+x_{1} x_{2}+\Delta f(x, t)+u+d(x, t),
\end{aligned}
$$

where $\Delta f(x, t)=0.39 \sin \left(x_{1} x_{2}+x_{3} \sqrt{t}\right)$ and $d(x, t)=$ $0.6 \sin (10 t)$. The initial conditions are chosen as: $x(0)=\left[\begin{array}{lll}-1 & 1.5 & 1\end{array}\right]^{T}$. The constant parameters are chosen as: $k=2, \beta=1, \kappa=1, \lambda=10, \mu=2, \gamma=3$, $\varepsilon=1, p_{0}=3, q_{0}=5$, and $\eta=\frac{3}{5}$. The trajectories of the states $x_{1}, x_{2}$, and $x_{3}$ are demonstrated in Figure 1. The time response of the control signal is demonstrated in Figure 2. It is shown that the suggested method can obtain superior performance and high robustness and is capable to control the parametric uncertainties and system nonlinearities. The time responses of the auxiliary variable $s$ and the NFTSMC surface $\sigma$ are demonstrated in Figure 3. Clearly, in can be seen that these sliding curves approach zero quickly. These numerical simulations approve the proposed technique.

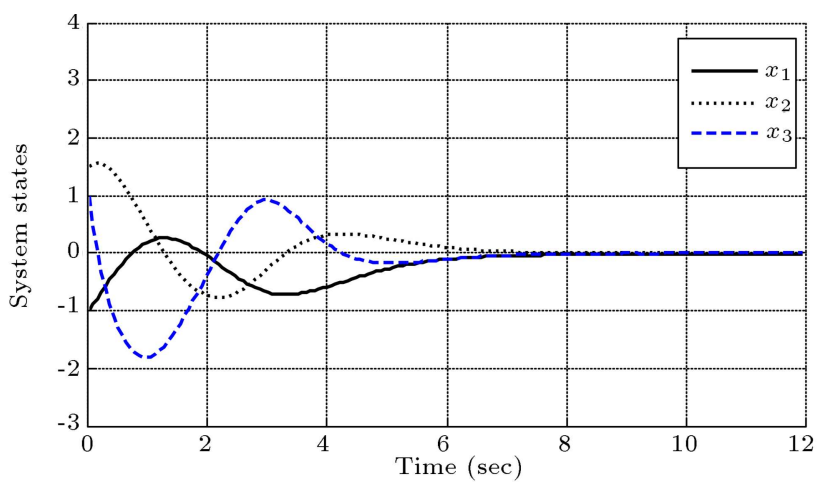

Figure 1. State trajectories

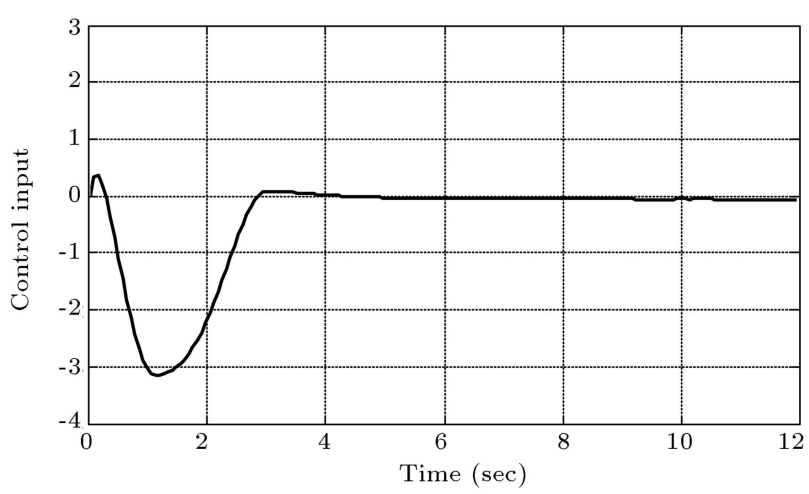

Figure 2. Control input $u$.

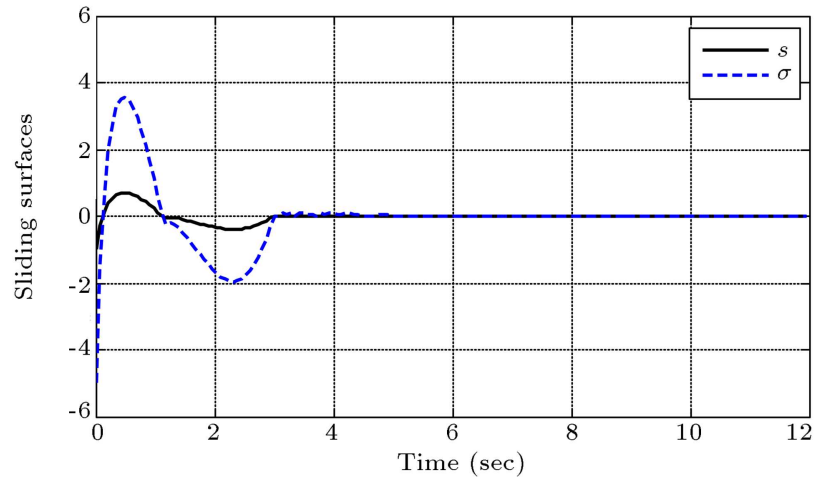

Figure 3. Sliding surfaces $s$ and $\sigma$.

Example 2. Consider the Lur'e-like chaotic system with an additive control input defined by [40]:

$$
\begin{aligned}
& \dot{x}_{1}=x_{2}, \\
& \dot{x}_{2}=x_{3}, \\
& \dot{x}_{3}=-6.8 x_{1}-3.9 x_{2}-x_{3}+12 \mathcal{X}\left(x_{1}\right)-0.8 \cos (5 t)+u,
\end{aligned}
$$

where:

$$
\mathcal{X}\left(x_{1}\right)= \begin{cases}P x_{1} & \text { if }\left|x_{1}\right|<\frac{1}{P} \\ \operatorname{sgn}\left(x_{1}\right) & \text { if }\left|x_{1}\right| \geq \frac{1}{P}\end{cases}
$$

System (34) represents a chaotic behavior for $P=1.5$. This chaotic system is numerically simulated using the offered control law with the following initial parameters and initial conditions:

$$
\begin{aligned}
& k=2, \quad \beta=1.5, \quad \kappa=2, \quad \lambda=8, \quad \mu=2, \quad \varepsilon=1, \\
& p_{0}=3, \quad q_{0}=5, \quad \eta=\frac{3}{5}, \quad x(0)=\left[\begin{array}{lll}
0.5 & 1 & 1
\end{array}\right]^{T} .
\end{aligned}
$$

The tracking trajectories of states $x_{1}, x_{2}$, and $x_{3}$ are shown in Figure 4. It is demonstrated in these figures that all of the states are stabilized. The dynamic

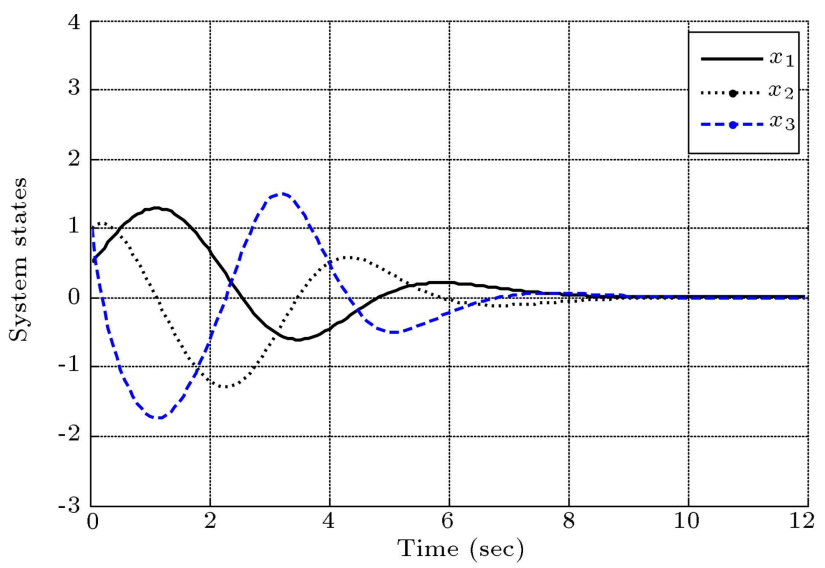

Figure 4. State trajectories. 


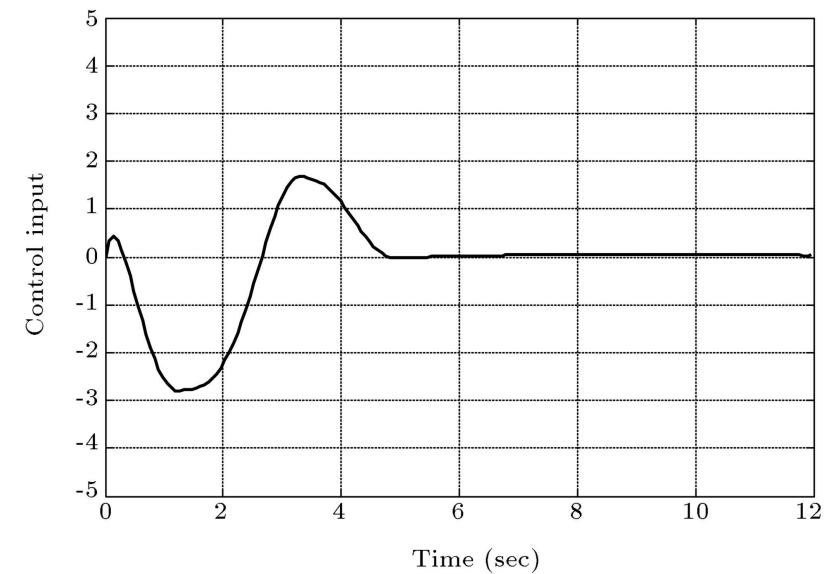

Figure 5. Control input $u$.

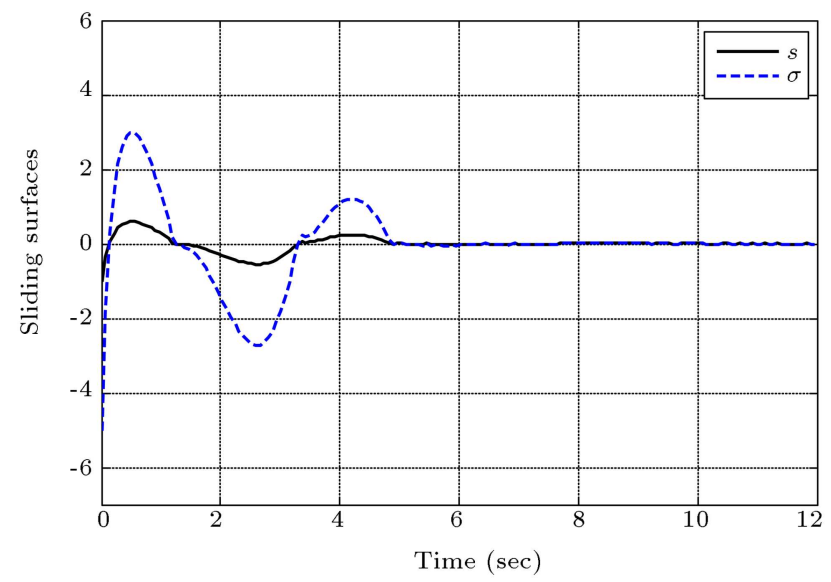

Figure 6. Sliding surfaces $s$ and $\sigma$.

control input, $u$, is demonstrated in Figure 5. The time responses of $s$ and $\sigma$ are shown in Figure 6. Obviously, it can be found that the switching surface and NFTSMC surface converge to the origin quickly. Therefore, the uncertain chaotic system is stabilized by applying the planned controller.

\section{Conclusions}

A new disturbance-observer-based finite-time stabilizer for the uncertain and nonlinear third-order systems is considered in this work. A novel reaching law is suggested to assure the presence of the switching behavior around the designed NFTSMC curve in the finite time. Moreover, in order to dominate the singularity phenomenon of FTSMC, a nonsingular control approach is proposed. Intensive numerical simulation results are displayed to confirm the effectiveness of the suggested technique and acceptable results are obtained. It is pointed out that the suggested approach can be employed for the tracking control and stabilization of higher-order uncertain and nonlinear dynamical systems.

\section{Acknowledgement}

This research project was supported by a grant from the "Research Center of the Center for Female Scientific and Medical Colleges", Deanship of Scientific Research, King Saud University.

\section{References}

1. Bhat, S.P. and Bernstein, D.S. "Continuous finite-time stabilization of the translational and rotational double integrators", IEEE Trans. Autom. Control, 43, pp. 678-682 (1998).

2. Liu, L., Fu, Z., Cai, X. and Song, X. "Non-fragile sliding mode control of discrete singular systems", Commun. Nonlinear Sci. Numer. Simul., 18, pp. 735743 (2013).

3. Li, T.H.S. and Huang, Y.C. "MIMO adaptive fuzzy terminal sliding-mode controller for robotic manipulators", Inform. Sci., 180, pp. 4641-4660 (2010).

4. Ou, M., Du, H. and Li, S. "Finite-time tracking control of multiple nonholonomic mobile robots", J. Franklin Instit., 349, pp. 2834-2860 (2012).

5. Mobayen, S. "Finite-time tracking control of chainedform nonholonomic systems with external disturbances based on recursive terminal sliding mode method", Nonlinear Dyn., 80, pp. 669-683 (2015).

6. Dadras, S. and Momeni, H.R. "Fractional-order dynamic output feedback sliding mode control design for robust stabilization of uncertain fractional-order nonlinear systems", Asian J. Control, 16, pp. 489-497 (2014).

7. Mobayen, S. "An LMI-based robust controller design using global nonlinear sliding surfaces and application to chaotic systems", Nonlinear Dyn., 79, pp. 10751084 (2014).

8. Mobayen, S. and Tchier, F. "Design of an adaptive chattering avoidance global sliding mode tracker for uncertain non-linear time-varying systems", Trans. Instit. Meas. Control (2016). DOI:10.1177/0142331216644046

9. Mobayen, S. "Design of LMI-based sliding mode controller with an exponential policy for a class of underactuated systems", Complexity, 21(5), pp. 117-124 (2014). DOI:10.1002/cplx.21636

10. Dong, L. and Tang, W.C. "Adaptive backstepping sliding mode control of flexible ball screw drives with timevarying parametric uncertainties and disturbances", ISA Trans., 53, pp. 110-116 (2014).

11. Mobayen, S. and Tchier, F. "A new LMI-based robust finite-time sliding mode control strategy for a class of uncertain nonlinear systems", Kybernetika, 51, pp. 1035-1048 (2015).

12. Mobayen, S. "An adaptive chattering-free PID sliding mode control based on dynamic sliding manifolds for a class of uncertain nonlinear systems", Nonlinear Dyn., 82, pp. 53-60 (2015). 
13. Mobayen, S. and Tchier, F. "An LMI approach to adaptive robust tracker design for uncertain nonlinear systems with time-delays and input nonlinearities", Nonlinear Dyn., 85(3), pp. 1965-1978 (2016).

14. Mobayen, S. "A novel global sliding mode control based on exponential reaching law for a class of under-actuated systems with external disturbances", J. Comput. Nonlinear Dyn., 11(2), p. 021011 (2015).

15. Young, K.D., Utkin, V.I. and Ozguner, U. "A control Engineer's guide to sliding mode control", IEEE Trans. Control Syst. Technol., 7(3), pp. 328-342 (1999).

16. Edwards, C. and Spurgeon, S., Sliding Mode Control: Theory and Applications, Taylor and Francis, London, UK (1998).

17. Pukdeboon, C. and Siricharuanun, P. "Nonsingular terminal sliding mode based finite-time control for spacecraft attitude tracking", Int. J. Control, Autom. Syst., 12, pp. 530-540 (2014).

18. Yang, J., Li, S., Su, J. and Yu, X. "Continuous nonsingular terminal sliding mode control for systems with mismatched disturbances", Automatica, 49, pp. 2287-2291 (2013).

19. Feng, Y., Yu, X. and Man, Z. "Non-singular terminal sliding mode control of rigid manipulators", Automatica, 38, pp. 2159-2167 (2002).

20. Yu, X. and Man, Z. "Fast terminal sliding mode control design for nonlinear dynamical systems", IEEE Trans. Circuit Syst. I Fundam. Theory Appl., 49(2), pp. 261264 (2002).

21. Mobayen, S., Yazdanpanah, M.J. and Majd, V.J. "A finite-time tracker for nonholonomic systems using recursive singularity-free FTSM", 2011 American Control Conference, San Francisco, CA, pp. 1720-1725 (2011).

22. Chen, M., Wu, Q.X. and Cui, R.X. "Terminal sliding mode tracking control for a class of SISO uncertain nonlinear systems", ISA Trans., 52, pp. 198-206 (2013).

23. Xu, Y., Wang, H., Liu, D. and Huang, H. "Sliding mode control of a class of fractional chaotic systems in the presence of parameter perturbations", J. Vib. Control (2013). DOI:1077546313486283

24. Chen, W.H. "Disturbance observer based control for nonlinear systems", IEEE Trans. Mechatron., 9, pp. 706-710 (2004).

25. Chen, M. and Chen, W.H. "Sliding mode controller design for a class of uncertain nonlinear system based disturbance observer", Int. J. Adapt. Control Signal Process., 24, pp. 51-64 (2010).

26. Ginoya, D., Shendge, P.D. and Phadke, S.B. "Disturbance observer based sliding mode control of nonlinear mismatched uncertain systems", Commun. Nonlinear Sci. Numer. Simul., 26, pp. 98-107 (2015).

27. Estrada, A., Loria, A., Santiesteban, R. and Fridman, L. "Cascaded-based stabilization of time-varying systems using second-order sliding modes", IMA J. Math. Control Inf., 30, pp. 115-128 (2013).
28. Mobayen, S. "Fast terminal sliding mode controller design for nonlinear second-order systems with timevarying uncertainties", Complexity, 21(2), pp. 239-244 (2015).

29. Mobayen, S. "Fast terminal sliding mode tracking of non-holonomic systems with exponential decay rate", IET Control Theory Appl., 9, pp. 1294-1301 (2015).

30. Mobayen, S. and Javadi, S. "Disturbance observer and finite-time tracker design of disturbed third-order nonholonomic systems using terminal sliding mode", J. Vib. Control, 23(2), pp. 181-189 (2017).

31. Mobayen, S. "An adaptive fast terminal sliding mode control combined with global sliding mode scheme for tracking control of uncertain nonlinear third-order systems", Nonlinear Dyn., 82, pp. 599-610 (2015).

32. Mobayen, S., Baleanu, D. and Tchier, F. "Secondorder fast terminal sliding mode control design based on LMI for a class of non-linear uncertain systems and its application to chaotic systems", J. Vib. Control (2016). DOI:10.1177/1077546315623887

33. Xiang, W. and Chen, F. "Robust synchronization of a class of chaotic systems with disturbance estimation", Commun. Nonlinear Sci. Numer. Simul., 16, pp. 29702977 (2011).

34. Kim, E. "A fuzzy disturbance observer and its application to control", IEEE Trans. Fuzzy Syst, 10, pp. 77-84 (2002).

35. Wei, X.J., Zhang, H.F. and Guo, L., "Composite disturbance-observer-based control and terminal sliding mode control for uncertain structural systems", Int. J. Syst. Sci., 40, pp. 1009-1017 (2009).

36. Wei, X. and Guo, L. "Composite disturbance-observerbased control and terminal sliding mode control for non-linear systems with disturbances", Int. J. Control, 82, pp. 1082-1098 (2009).

37. Zhang, R., Sun, C., Zhang, J. and Zhou, Y. "Secondorder terminal sliding mode control for hypersonic vehicle in cruising flight with sliding mode disturbance observer", J. Control Theory Appl., 11, pp. 299-305 (2013).

38. Chang, J.L. "Dynamic output feedback integral sliding mode control design for uncertain systems", Int. J. Robust Nonlinear Control, 22, pp. 841-857 (2012).

39. Bartoszewicz, A. and Nowacka, A. "Sliding-mode control of the third-order system subject to velocity, acceleration and input signal constraints", Int. J. Adapt. Control Signal Process., 21, pp. 779-794 (2007).

40. Feki, M. "An adaptive feedback control of linearizable chaotic systems", Chaos, Soliton. Fract., 15, pp. 883890 (2003).

\section{Biographies}

Saleh Mobayen received his doctorate in Control Engineering from Tarbiat Modares University, Iran, in 2012, and is currently Assistant Professor of Control 
Engineering at University of Zanjan. He has published several papers in the national and international journals. His research interests include sliding-mode control, robust tracking, non-holonomic robots, and chaotic systems.

Fairouz Tchier received her $\mathrm{PhD}$ degree in Math- ematics with the specialty of Theoretical Computer Science from Université Laval, Quebec City, Québec, Canada, in 1996, and is currently an Associate Professor in Mathematics Department, King Saud University. Her research interests include theoretical computer science, software engineering, fixed point theory, formal methods, and fuzzy set theory. 